\title{
Detection of mild cognitive impairment in middle-aged and older adults with obstructive sleep apnoea
}

\author{
Katia Gagnon ${ }^{1,2}$, Andrée-Ann Baril ${ }^{1,3}$, Jacques Montplaisir ${ }^{1,3}$, Julie Carrier ${ }^{1,4}$, \\ Sirin Chami ${ }^{1,3}$, Serge Gauthier ${ }^{5}$, Chantal Lafond ${ }^{1}$, Jean-François Gagnon ${ }^{1,2}$ and \\ Nadia Gosselin ${ }^{1,4}$
}

Affiliations: ${ }^{1}$ Centre for Advanced Research in Sleep Medicine (CARSM), Hôpital du Sacré-Coeur de Montréal, Montreal, QC, Canada. ${ }^{2}$ Dept of Psychology, Université du Québec à Montréal, Montreal, QC, Canada. ${ }^{3}$ Dept of Psychiatry, Université de Montréal, Montreal, QC, Canada. ${ }^{4}$ Dept of Psychology, Université de Montréal, Montreal, QC, Canada. ${ }^{5}$ Dept of Psychiatry, Neurology and Neurosurgery, McGill University, Montreal, QC, Canada.

Correspondence: Nadia Gosselin, Center for Advanced Research in Sleep Medicine, Hôpital du Sacré-Cœur de Montréal, 5400 Gouin Blvd West, Room J-5135, Montreal, Quebec, H4J 1C5, Canada.

E-mail: nadia.gosselindumontreal.ca

@ERSpublications

The Montreal Cognitive Assessment performs better than the Mini-Mental State Examination in detecting cognitive impairment in individuals with obstructive sleep apnoea http://ow.ly/8gLS30lxjZk

Cite this article as: Gagnon K, Baril A-A, Montplaisir J, et al. Detection of mild cognitive impairment in middle-aged and older adults with obstructive sleep apnoea. Eur Respir J 2018; 52: 1801137 [https://doi. org/10.1183/13993003.01137-2018].

ABSTRACT Obstructive sleep apnoea increases the risk for mild cognitive impairment and dementia. The present study aimed to characterise the ability of two cognitive screening tests, the Mini-Mental State Examination and the Montreal Cognitive Assessment, to detect mild cognitive impairment in adults aged 55-85 years with and without obstructive sleep apnoea.

We included 42 subjects with mild and 67 subjects with moderate-to-severe obstructive sleep apnoea. We compared them to 22 control subjects. Mild cognitive impairment was diagnosed by a comprehensive neuropsychological assessment. We used receiver operating characteristic curves to assess the ability of the two screening tests to detect mild cognitive impairment.

The two screening tests showed similar discriminative ability in control subjects. However, among the mild and the moderate-to-severe obstructive sleep apnoea groups, the Mini-Mental State Examination was not able to correctly identify subjects with mild cognitive impairment. The Montreal Cognitive Assessment's discriminant ability was acceptable in both sleep apnoea groups and was comparable to what was observed in controls.

The Mini-Mental State Examination should not be used to screen for cognitive impairment in patients with obstructive sleep apnoea. The Montreal Cognitive Assessment could be used in clinical settings. However, clinicians should refer patients for neuropsychological assessment when neurodegenerative processes are suspected. 


\section{Introduction}

Recent studies have shown that obstructive sleep apnoea (OSA) is a risk factor for mild cognitive impairment (MCI) and dementia in older adults [1-4]. Being able to easily identify patients at risk of cognitive decline could significantly affect treatment decisions for OSA and modify the course of their clinical follow-up, particularly in patients presenting with no or mild daytime sleepiness. Clinicians can screen for cognitive decline using patients' subjective report or objective validated cognitive tests. Although subjective cognitive complaint is a good predictor of MCI and dementia in the elderly [5-8], this method is not appropriate for OSA patients. In fact, $23-70 \%$ of OSA patients report subjective cognitive complaints, but most of the time these complaints are not associated with an objective impairment [9]. This suggests that asking OSA patients to evaluate their cognitive status might not reflect objective cognitive decline, which underscores the importance of using screening tests to detect cognitive impairment in this population.

The Mini-Mental State Examination (MMSE) and the Montreal Cognitive Assessment (MoCA) are cognitive screening tests widely used to identify MCI in older adults $[10,11]$. Studies performed in elderly individuals indicate that the MoCA is a better discriminative tool for detecting MCI than the MMSE [1215]. However, the MoCA seems less able to detect MCI in populations with comorbidities (e.g. Parkinson's disease, chronic obstructive pulmonary disease) than in populations with no comorbid conditions (area under the curve (AUC) of $0.79-0.84$ versus $0.85-0.90$, respectively) $[11,16-18]$. The same pattern has been reported for MMSE $[12-14,17,18]$. These observations raise questions regarding the ability of cognitive screening tests to detect MCI in clinical populations, including OSA.

The present study aimed to compare the validity of the MMSE and the MoCA in detecting MCI in individuals $\geqslant 55$ years without (control subjects) or with OSA (mild versus moderate-to-severe). Studies have consistently shown that the MoCA is superior to the MMSE in detecting MCI [12-14, 16-18], and the Beijing version of the MoCA has been shown to reliably detect cognitive dysfunction in young OSA patients [19]. Therefore, we hypothesised that the MoCA would be better at identifying MCI in the three groups. We also predicted that the MoCA and the MMSE would have lower discriminant validity in subjects with mild or moderate-to-severe OSA compared to in control participants [12-14].

In order to clarify whether discrepancies in the validity of screening tests could be caused by the different nature of cognitive deficits in mild OSA, moderate-to-severe OSA and control subjects, we also compared groups based on their MCI subtypes (amnestic versus non-amnestic; single versus multiple cognitive domains impaired). We hypothesised that control participants with MCI would have a cognitive profile typical of preclinical Alzheimer's disease, with a predominance of amnestic MCI [20]. However, we expected that more heterogeneous cognitive profiles would be found in patients with mild and moderate-to-severe OSA because their cognitive impairments may not be entirely due to neurodegeneration [21-24].

\section{Methods}

Detailed methods are described in the supplementary material. A total of 131 participants were recruited for this study from 2012 to 2017; 76\% of these participants have been included in previous articles on genetics and neuroimaging [25-27]. The recruitment procedure and inclusion/exclusion criteria were extensively described in previous studies [25-27]. Briefly, we included subjects aged 55-85 years with $>7$ years of education. We excluded participants with a diagnosis of morbid obesity, neurological disease (e.g. Parkinson's disease, previous stroke, brain tumour, epilepsy), psychiatric disease (e.g. diagnosed major depression and anxiety disorder), a sleep disorder other than OSA or a respiratory disorder other than OSA (e.g. chronic obstructive pulmonary disease), or who were prescribed medication that may affect cerebral functioning, such as hypnotics, antidepressants, anticonvulsants or opioids. The hospital ethics committee approved the study and all participants gave their written informed consent to participate in the study.

\section{Protocol overview}

All participants filled out questionnaires regarding their sleep quality and mood. They underwent one night of in-laboratory polysomnography recording that included electroencephalography, electromyography (chin and anterior tibialis), electro-oculography and electrocardiography as well as measurements from thoraco-abdominal strain gauges, an oronasal cannula and a transcutaneous finger pulse oximeter.

The next morning, all participants underwent a 3-h neuropsychological assessment with tests administered in the same order and according to standard procedures [28]. The MoCA and the MMSE were administered first. Detailed procedures for administration and scoring of screening tests and the complete 
neuropsychological evaluation procedure are detailed in the supplementary methods and supplementary table E1.

We used the three following criteria for MCI diagnosis [29]: 1) an objective cognitive impairment (score $\leqslant 1.5 \mathrm{SD}$ ) on at least two measures of the same cognitive domain (attention and speed processing; executive functions; visual and verbal episodic learning and memory; visuospatial abilities; language); 2) preserved independence in daily activities according to the Activities of Daily Living Inventory [30]; and 3) psychiatric condition or medication use cannot better explain the presence of cognitive impairments. We categorised MCI according to four subtypes: amnestic single domain, amnestic multiple domains, non-amnestic single domain and non-amnestic multiple domains [29].

\section{Statistical analyses}

We divided participants based on their apnoea-hypopnoea index (AHI). Subjects with AHI $<5$ events $\cdot \mathrm{h}^{-1}$ were considered as controls; AHI $\geqslant 5$ events $\cdot \mathrm{h}^{-1}$ and $<15$ events $\cdot \mathrm{h}^{-1}$ were classed as having mild OSA; and $\mathrm{AHI} \geqslant 15$ events. $\mathrm{h}^{-1}$ were classed as having moderate-to-severe OSA [31]. Participants were also divided according to their cognitive status (non-MCI versus MCI). We performed group (control, mild OSA and moderate-to-severe OSA) by cognitive status (non-MCI and MCI) ANOVAs on demographic and clinical variables. We decomposed significant interactions using simple effect analysis.

We used receiver operating characteristic (ROC) curve analyses with area under the curve (AUC) and 95\% CI to determine the MoCA and the MMSE discriminant indices in MCI detection (i.e. sensitivity, specificity, negative predictive value, positive predictive value and percentage correctly categorised) in each group separately. We used Youden's index ( $y=$ sensitivity + specificity-1) combined with examination of the balance between sensitivity and specificity to determine optimal cut-off values. Discriminative abilities of the MoCA and the MMSE were compared between groups using the method of HANLEY and McNeIL [32]. Pearson's Chi-squared tests were used to compare groups for MCI subtypes. We performed statistical analyses with SPSS for Mac 20.0 (SPSS Science, Chicago, IL, USA). Statistical significance was set at $\mathrm{p}<0.05$.

\section{Results}

\section{Sample characteristics}

The sample included non-OSA and OSA participants with a wide range of severity (AHI 0-84 events $\mathrm{h}^{-1}$ ). Group characteristics and statistics are outlined in table 1 . We recruited a similar proportion of patients with MCI in all groups (control: 36.4\%; mild OSA: 40.5\%; moderate-to-severe OSA: 40.3\%), allowing between-group comparisons with equivalent cognitive status. No significant differences were found among the four groups for age, sex or education. We found no significant group-by-cognitive status interactions for demographic variables. However, a cognitive status effect (MCI versus non-MCI) was found for education, with MCI participants having fewer years of education than non-MCI participants $(F(1125)=12.6 ; \mathrm{p}<0.01)$.

\section{Questionnaires}

We found that the moderate-to-severe OSA group had a significantly higher score on the Pittsburgh Sleep Quality Index than the control group (table 1). No significant interactions or other group effects were found for the questionnaires.

\section{OSA-related variables}

We found no significant group-by-cognitive status interactions on polysomnographic variables. However, we observed expected group differences for OSA versus control participants, more specifically for body mass index, sleep fragmentation, percentage of rapid eye movement (REM) sleep and oxygen saturation (table 1).

Detection of $\mathrm{MCl}$ in participants with moderate-to-severe OSA using screening tests ROC curve analyses are presented in table 2 and illustrated in figure 1a. The MMSE mean \pm SD score was $28.64 \pm 1.32$ for participants with moderate-to-severe OSA, and ROC curve analysis showed an AUC of 0.64 (95\% CI 0.51-0.77). The optimal MMSE cut-off value was 30 ( $\leqslant 29$ indicating MCI with $89 \%$ sensitivity, $43 \%$ specificity and $61 \%$ correctly classified). The MoCA mean \pm SD score in participants with moderate-to-severe OSA was 26.48 \pm 2.48 , and ROC curve analysis showed an AUC of 0.82 (95\% CI $0.71-$ 0.92). The optimal MoCA cut-off value was 27 ( $\leqslant 26$ indicating MCI with $70 \%$ sensitivity, $73 \%$ specificity and $72 \%$ correctly classified). 
TABLE 1 Demographic and clinical characteristics of control participants and patients with mild obstructive sleep apnoea (OSA) and moderate-to-severe OSA

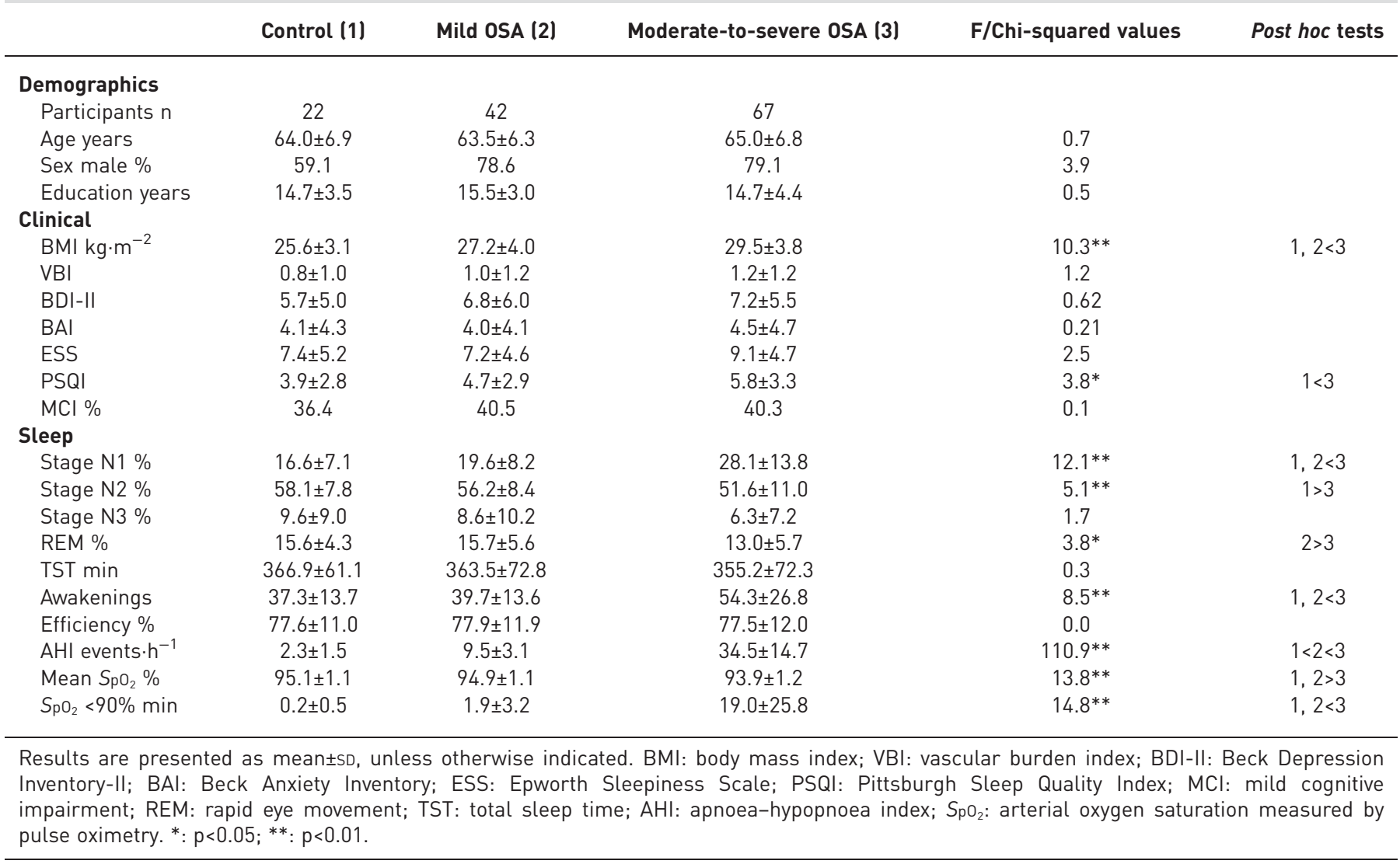

Detection of $\mathrm{MCl}$ in participants with mild OSA using screening tests

The mean \pm SD score of the MMSE was $28.86 \pm 1.35$ for participants with mild OSA, and ROC curve analysis showed an AUC of 0.74 (95\% CI 0.59-0.90). The MMSE optimal cut-off was 28 ( $\leqslant 27$ indicating MCI with $53 \%$ sensitivity, $84 \%$ specificity and $71 \%$ correctly classified). The MoCA mean \pm sD score was $27.02 \pm 2.23$, and ROC curve analysis showed an AUC of 0.85 (95\% CI 0.74-0.97) (figure 1b). The optimal MoCA cut-off was 28 ( $\leqslant 27$ indicated MCI with $88 \%$ sensitivity, $76 \%$ specificity and $91 \%$ correctly classified) (table 3).

TABLE 2 Validity of the MoCA and the MMSE in detecting mild cognitive impairment in participants with moderate-to-severe obstructive sleep apnoea

\begin{tabular}{|c|c|c|c|c|c|c|c|c|c|c|c|}
\hline \multicolumn{6}{|c|}{ МoCA } & \multicolumn{6}{|c|}{ MMSE } \\
\hline Cut-off & Sens. & Spec. & PPV & NPV & $\%$ Correct & Cut-off & Sens. & Spec. & PPV & NPV & $\%$ Correct \\
\hline $30 / 29$ & 100 & 18 & 45 & 100 & 51 & $30 / 29^{\#}$ & 89 & 43 & 51 & 85 & 61 \\
\hline $29 / 28$ & 96 & 33 & 49 & 93 & 58 & $29 / 28$ & 44 & 65 & 46 & 63 & 57 \\
\hline $28 / 27$ & 89 & 55 & 57 & 88 & 69 & $28 / 27$ & 22 & 90 & 60 & 63 & 63 \\
\hline $27 / 26^{\#}$ & 70 & 73 & 63 & 78 & 72 & $27 / 26$ & 7 & 93 & 40 & 60 & 58 \\
\hline $26 / 25$ & 59 & 85 & 73 & 76 & 75 & $26 / 25$ & 4 & 98 & 50 & 60 & 60 \\
\hline $25 / 24$ & 37 & 98 & 91 & 70 & 73 & $25 / 24$ & 4 & 100 & 100 & 61 & 61 \\
\hline $24 / 23$ & 26 & 98 & 88 & 66 & 67 & $24 / 23$ & 0 & 100 & 0 & 60 & 60 \\
\hline $23 / 22$ & 11 & 100 & 100 & 63 & 64 & $23 / 22$ & 0 & 100 & 0 & 60 & 60 \\
\hline \multicolumn{6}{|c|}{$\operatorname{AUC}(95 \% \mathrm{CI}): 0.82(0.71-0.92)$} & \multicolumn{6}{|c|}{$\operatorname{AUC}(95 \% \mathrm{CI}): 0.64(0.51-0.77)$} \\
\hline
\end{tabular}

MoCA: Montreal Cognitive Assessment; MMSE: Mini-Mental State Examination; Sens.: sensitivity; Spec.: specificity; PPV: positive predictive value; NPV: negative predictive value; AUC: area under the curve. ${ }^{\#}$ : optimal cut-off. 
a)

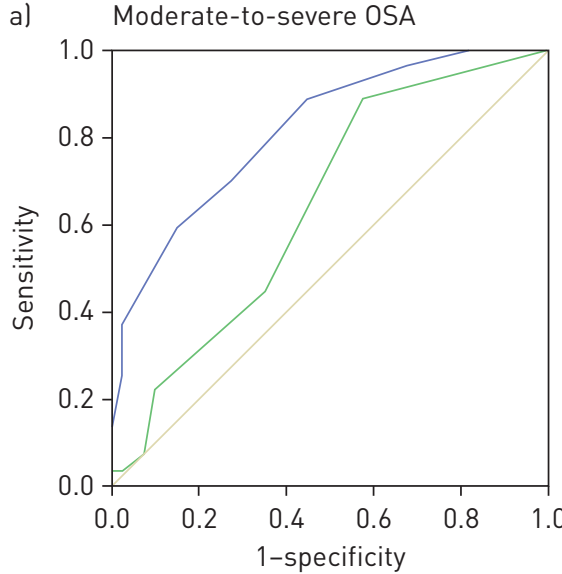

b)

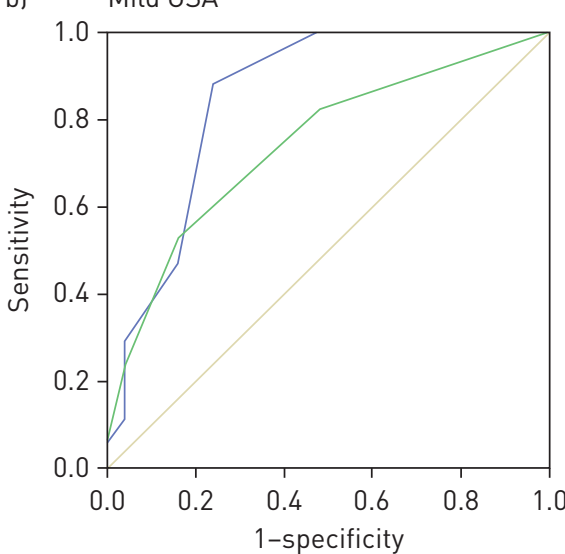

c)

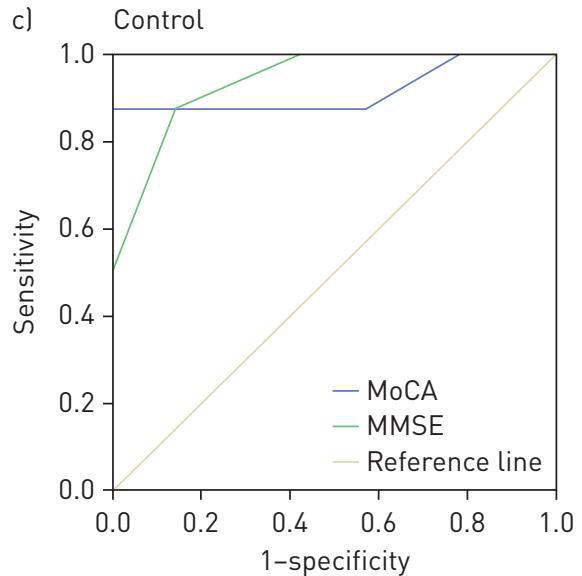

FIGURE 1 Receiver operating characteristic (ROC) curve comparisons between the Montreal Cognitive Assessment (MoCA) and the Mini-Mental State Examination (MMSE) in detecting mild cognitive impairment (MCI) in a) moderate-to-severe obstructive sleep apnoea (OSA), b) mild OSA and c) control participants. a) Participants with moderate-to-severe OSA had a significantly higher area under the curve (AUC) for the MoCA (AUC 0.82, 95\% Cl 0.71-0.92) than for the MMSE (AUC 0.64, 95\% Cl 0.51-0.77). b) For participants with mild OSA, the ROC curve had higher AUC for the MoCA (AUC 0.85, 95\% Cl 0.74-0.97) than for the MMSE (AUC 0.74, 95\% Cl 0.59-0.90). c) Among control participants, the ROC curve had a similar AUC for the MoCA (AUC 0.92, 95\% Cl 0.75-1.00) and the MMSE (AUC 0.94, 95\% Cl 0.84-1.00).

\section{Detection of $\mathrm{MCl}$ in control participants using screening tests}

The MMSE mean \pm SD score was $28.36 \pm 1.97$ for control participants, and the ROC curve analysis showed an AUC of 0.94 (95\% CI 0.84-1.00). The optimal MMSE cut-off was 29 ( $\leqslant 28$ indicating MCI with $88 \%$ sensitivity, $87 \%$ specificity and $86 \%$ correctly classified). The MoCA mean \pm SD score was $26.45 \pm 3.10$, and ROC curve analysis showed an AUC of 0.92 (95\% CI 0.78-1.00) (figure 1c). The optimal MoCA cut-off was 27 ( $\leqslant 26$ indicated MCI with $88 \%$ sensitivity, $86 \%$ specificity and $86 \%$ correctly classified) (table 4 ).

\section{Between-group comparisons for discriminant validity}

When we compared the discriminant ability of the screening tests between groups using the method of HANLEY and MCNeIL [32], we found that the MMSE showed significantly higher discriminant validity in the control group than in the moderate-to-severe OSA group (see the tables for AUC and confidence intervals; $\mathrm{p}<0.01)$ and a trend for higher discriminant validity in the control group compared to the mild OSA group $(\mathrm{p}=0.05)$. There were no significant differences in the MoCA's discriminant abilities between control subjects and participants with mild or moderate-to-severe OSA ( $p=0.23$ and $p=0.13$, respectively).

Cognitive profile in OSA versus non-OSA participants

The control group showed a higher percentage of amnestic MCI (87.5\%) than the mild OSA group $(64.7 \%)$ and the moderate-to-severe OSA group (59.3\%); however, this difference was nonsignificant

TABLE 3 Validity of the MoCA and the MMSE in detecting mild cognitive impairment in participants with mild obstructive sleep apnoea

\begin{tabular}{|c|c|c|c|c|c|c|c|c|c|c|c|}
\hline \multicolumn{6}{|c|}{ MoCA } & \multicolumn{6}{|c|}{ MMSE } \\
\hline Cut-off & Sens. & Spec. & PPV & NPV & $\%$ Correct & Cut-off & Sens. & Spec. & PPV & NPV & $\%$ Correct \\
\hline $30 / 29$ & 100 & 24 & 47 & 100 & 55 & $30 / 29$ & 82 & 52 & 54 & 81 & 64 \\
\hline $29 / 28$ & 100 & 52 & 59 & 100 & 71 & $29 / 28^{\#}$ & 53 & 84 & 69 & 72 & 71 \\
\hline $28 / 27^{\#}$ & 88 & 76 & 71 & 91 & 81 & $28 / 27$ & 24 & 96 & 80 & 65 & 67 \\
\hline $27 / 26$ & 71 & 80 & 71 & 80 & 76 & $27 / 26$ & 6 & 100 & 100 & 61 & 62 \\
\hline $26 / 25$ & 47 & 84 & 67 & 70 & 69 & $26 / 25$ & 6 & 100 & 100 & 64 & 62 \\
\hline $25 / 24$ & 29 & 96 & 83 & 67 & 69 & $25 / 24$ & 6 & 100 & 100 & 61 & 62 \\
\hline $24 / 23$ & 12 & 96 & 67 & 62 & 62 & $24 / 23$ & 6 & 100 & 100 & 61 & 62 \\
\hline $23 / 22$ & 6 & 100 & 100 & 61 & 62 & $23 / 22$ & 0 & 100 & 0 & 60 & 60 \\
\hline \multicolumn{6}{|c|}{$\operatorname{AUC}(95 \% \mathrm{Cl}): 0.85(0.74-0.97)$} & \multicolumn{6}{|c|}{$\operatorname{AUC}(95 \% \mathrm{CI}): 0.74(0.59-0.90)$} \\
\hline
\end{tabular}

MoCA: Montreal Cognitive Assessment; MMSE: Mini-Mental State Examination; Sens.: sensitivity; Spec.: specificity; PPV: positive predictive value; NPV: negative predictive value; AUC: area under the curve. ${ }^{\#}$ : optimal cut-off. 
TABLE 4 Validity of the MoCA and the MMSE in detecting mild cognitive impairment in control participants

\begin{tabular}{|c|c|c|c|c|c|c|c|c|c|c|c|}
\hline \multicolumn{6}{|c|}{ MoCA } & \multicolumn{6}{|c|}{ MMSE } \\
\hline Cut-off & Sens. & Spec. & PPV & NPV & $\%$ Correct & Cut-off & Sens. & Spec. & PPV & NPV & $\%$ Correct \\
\hline $30 / 29$ & 100 & 21 & 42 & 100 & 50 & $30 / 29$ & 100 & 57 & 57 & 100 & 73 \\
\hline $29 / 28$ & 88 & 43 & 47 & 86 & 59 & $29 / 28^{\# \#}$ & 88 & 87 & 78 & 92 & 86 \\
\hline $28 / 27$ & 88 & 79 & 70 & 92 & 82 & $28 / 27$ & 50 & 100 & 100 & 78 & 82 \\
\hline $27 / 26$ & 88 & 86 & 78 & 92 & 86 & $27 / 26$ & 50 & 100 & 100 & 78 & 82 \\
\hline $26 / 25^{\#}$ & 88 & 100 & 100 & 93 & 95 & $26 / 25$ & 38 & 100 & 100 & 74 & 77 \\
\hline $25 / 24$ & 28 & 100 & 100 & 93 & 95 & $25 / 24$ & 13 & 100 & 100 & 67 & 68 \\
\hline $24 / 23$ & 63 & 100 & 100 & 82 & 86 & $24 / 23$ & 13 & 100 & 100 & 67 & 68 \\
\hline $23 / 22$ & 50 & 100 & 100 & 78 & 82 & $23 / 22$ & 0 & 100 & 0 & 64 & 64 \\
\hline \multicolumn{6}{|c|}{$\operatorname{AUC}(95 \% \mathrm{CI}): 0.92(0.75-1.00)$} & \multicolumn{6}{|c|}{$\operatorname{AUC}(95 \% \mathrm{CI}): 0.94(0.84-1.00)$} \\
\hline
\end{tabular}

MoCA: Montreal Cognitive Assessment; MMSE: Mini-Mental State Examination; Sens.: sensitivity; Spec.: specificity; PPV: positive predictive value; NPV: negative predictive value; AUC: area under the curve. ${ }^{\#}$ : optimal cut-off.

(Chi-squared (2)=2.18, $\mathrm{p}=0.34$ ). Surprisingly, groups did not differ regarding the proportion of single versus multiple cognitive domains affected (Chi-squared $(2)=0.99, p=0.61$ ) (supplementary figure E1).

\section{Discussion \\ Main findings}

This study aimed to evaluate the ability of two widely used cognitive screening tools, the MMSE and the MoCA, to detect MCI in OSA patients aged $\geqslant 55$ years compared to a group of non-OSA individuals. We found that the MoCA performed similarly to detect MCI in participants with mild and moderate-to-severe OSA and control subjects. More specifically, our results showed that the MoCA was able to correctly identify $81 \%$ of participants with mild OSA and $72 \%$ of participants with moderate-to-severe OSA who had MCI, whereas $86 \%$ of control subjects were correctly identified as having MCI. The optimal MoCA cut-off to discriminate MCI in mild OSA was $\leqslant 27$, and $\leqslant 26$ for participants with moderate-to-severe OSA, with very good AUC ( 0.85 and 0.82 , respectively) and acceptable sensitivity and specificity. Clinicians can therefore use the MoCA to screen for MCI in older adults with OSA, but they have to be aware that this screening test will correctly identify only $72 \%$ of their patients with moderate-to-severe OSA.

Regarding the MMSE in OSA participants, the more balanced MMSE cut-off for detecting MCI was $\leqslant 28$ for mild OSA and $\leqslant 29$ for moderate-to-severe OSA; therefore, only OSA patients with a score of 29 out of 30 or a perfect score of 30 out of 30 were considered to not present with MCI in our sample. This result indicates that the MMSE is not a valid screening tool to detect MCI in OSA patients. In fact, in patients with mild OSA, we found that the MMSE had poor sensitivity (53\%), good specificity ( $84 \%)$ and only a fair AUC (0.74), whereas in the moderate-to-severe OSA group the MMSE had good sensitivity (89\%) but very poor specificity (43\%) and unacceptable AUC (0.64) [33]. This screening tool should not be used for OSA patients in clinical settings, because it will result in a high number of undetected patients with MCI or false positives.

\section{Cognitive screening tests in patients with comorbid conditions}

Although the MoCA had better sensitivity and specificity than the MMSE, this screening test has to be used with caution in patients with OSA and those with comorbid clinical conditions. In fact, our results showed that the MoCA tended to perform more poorly in detecting MCI in mild and moderate-to-severe OSA compared with in our control participants, as well as in control subjects tested in previous studies $[11,16-18]$. We also observed lower discriminant validity for the MMSE in OSA participants compared to control participants. Our results therefore confirm previous observations that screening tests have lower validity in patients with a medical condition [11-18].

\section{Better discriminant ability of the MoCA to screen for cognitive impairment}

The higher discriminant ability observed for the MoCA compared to the MMSE in OSA patients is concordant with previous studies that found the MoCA better at detecting MCI than the MMSE [12-15, 17]. This higher discriminant validity could be explained by the fact that the two screening instruments do not cover the exact same cognitive domains [18]. The MMSE focuses on the evaluation of language and orientation, whereas the MoCA covers more broadly different cognitive domains, including attention and executive functions. In subjects with OSA and MCI, the cognitive profile may not be entirely attributable 
to ongoing neurodegenerative processes; it could also be due to chronic sleep fragmentation and intermittent hypoxaemia, which lead to attention, episodic memory and executive dysfunctions even in younger adults [21].

When we compared MCI subtypes between mild OSA, moderate-to-severe OSA and control participants, we found no significant differences for the type (amnestic versus non-amnestic) or number (single versus multiple) of cognitive domains impaired.

\section{Low MoCA score and risk of cognitive decline}

The prevalence of MCI in individuals with OSA is unknown but is suspected to be high given the common risk factors that OSA shares with MCI (apolipoprotein epsilon 4 allele, hypertension, obesity and metabolic syndrome) [34]. Moreover, sleep fragmentation and intermittent hypoxaemia secondary to OSA may directly increase the risk of abnormal cognitive decline by increasing amyloid depositions and tau hyperphosphorylation in the brain, two main mechanisms in Alzheimer's disease pathogenesis [35]. In the present study, we recruited our subjects based on their suspected or confirmed diagnosis of OSA at study entry; $40.5 \%$ of participants with mild OSA and $40.3 \%$ of individuals with moderate-to-severe OSA had MCI, but this proportion of MCI patients has to be confirmed in large cohort studies. Moreover, whether OSA patients with MCI have a higher risk than non-OSA adults of developing dementia is unknown. In previous retrospective studies using the MoCA to predict dementia, it was observed that $69.1 \%$ of MCI convert to dementia over a period of 18 months [36]. Longitudinal studies are needed to determine whether low MoCA scores reflect ongoing neurodegenerative processes and will predict progression to dementia in individuals with OSA.

\section{Strengths and limitations}

Our sample included a limited number of control subjects. However, when we compared the results obtained in our control subjects to those found in previous studies, we observed that the discriminant ability of the cognitive tests was similar for our control subjects and those included in the other studies $[11,16-18]$. Another weakness of the study was that we did not have longitudinal data that would have allowed a better understanding of the consequences of a low MoCA score over time, as well as the nature (i.e. neurodegenerative or not) of the cognitive impairment observed.

\section{Conclusion}

Our study showed that the MoCA is an effective screening tool for cognitive impairment in OSA. Although the MMSE remains widely used in clinical practice [18], our study found that this instrument could not acceptably detect MCI in patients with OSA. Because older OSA patients are more at risk of MCI and dementia, we suggest that clinicians should systematically use the MoCA to identify OSA patients with cognitive impairment and to track changes in their cognitive profile depending on whether they use an OSA treatment or not. However, the MoCA should be used with caution because between 19\% and $28 \%$ of our MCI participants with OSA were not detected by this screening tool. Consequently, clinicians should refer patients to a neuropsychologist when a significant cognitive decline is suspected, particularly when the patient is reluctant to use continuous positive airway pressure treatment or, in the case of milder forms of OSA, where the decision to treat or not depends more on daytime symptomatology.

Acknowledgements: We would like to thank Hélène Blais, Jean Paquet and Marie-Josée Quinn for their efforts and support in data acquisition and analyses. We would also like to thank neuropsychologists and graduate students in neuropsychology, Catherine Chapados, Marjolaine Lafortune, Frédérique Escudier, Jessica Cole and Johnathan Deslauriers, as well as the medical electrophysiology technologists Benoit Adam, Nancy Poirier, Mélina-Crécia Babala and Jean-Claude Aubertin. Finally, we would like to thank Maria Tuineag, Sarah Julien and Joëlle Robert for their assistance in polysomnographic recordings.

Author contributions: K. Gagnon contributed to the study's conception and design and to data acquisition, analysis and interpretation, and drafted the paper and revised it following author comments. A-A. Baril and S. Chami contributed to the study's design, data acquisition and interpretation, and to the critical revision of the work. J. Montplaisir, J. Carrier, C. Lafond, S. Gauthier and J-F. Gagnon contributed to the study's conception and to data interpretation, and revised the work critically. N. Gosselin contributed to the study's conception and design and to data interpretation, and also helped draft the paper and revised it critically. All authors approved the final version for publication and are accountable for all aspects of the work.

Conflict of interest: K. Gagnon has nothing to disclose. A-A. Baril reports government agency scholarships from Canadian Institutes of Health Research and Fonds de la Recherche du Québec-Santé, during the conduct of the study. J. Montplaisir reports personal fees for advisory board work from Novartis, Merck Pharmaceutical and Otsuka Pharmaceutical, outside the submitted work. J. Carrier reports grants to the Canadian Sleep and Circadian Network from RANA, Respironics and Merck, outside the submitted work. S. Chami has nothing to disclose. S. Gauthier reports government agency grants from Canadian Institutes of Health Research, during the conduct of the study; and grants 
from CQDM network, government agency grants from Pfizer-Fond de Recherche du Québec-Santé and Canadian Institutes of Health Research, personal fees for clinical trial support and scientific advisory work from Lilly, Roche and TauRx, personal fees for clinical trial support from Lundbeck, personal fees for scientific advisory work from AbbVie, ADvantage, Alzheon, Axovant, Boehringer Ingelheim, Firalis, Heptares, IntelGen, Klagene, Novartis, Otsuka, Servier, Sanofi, Schwabe and Takeda, and is a member of data and safety monitoring boards for the Alzheimer's Disease Cooperative Study and Alzheimer's Disease Therapeutic Research Institute, outside the submitted work. C. Lafond has nothing to disclose. J-F. Gagnon reports grants from W. Garfield Weston Foundation, Canadian Institutes of Health Research, Canada Research Chair and Fonds de Recherche du Québec-Santé, during the conduct of the study. N. Gosselin reports government agency grants from Canadian Institutes of Health Research, and government agency grants and salary awards from Fonds de Recherche du Québec-Santé, during the conduct of the study.

Support statement: This research was supported by the Canadian Institutes of Health Research (MOP123294) and by the Fonds de Recherche du Québec-Santé (FRQS). The Canadian Institutes of Health Research supported A-A. Baril with a doctoral scholarship and J-F. Gagnon with an Investigator Salary Award. The FRQS also supported N. Gosselin, J-F. Gagnon and J. Carrier with a Salary Award. J-F. Gagnon holds a Canada Research Chair in Cognitive Decline in Pathological Aging and J. Montplaisir holds a Canada Research Chair in Sleep Medicine.

\section{References}

1 Yaffe K, Laffan AM, Harrison SL, et al. Sleep-disordered breathing, hypoxia, and risk of mild cognitive impairment and dementia in older women. JAMA 2011; 306: 613-619.

2 Osorio RS, Gumb T, Pirraglia E, et al. Sleep-disordered breathing advances cognitive decline in the elderly. Neurology 2015; 84: 1964-1971.

3 Chang WP, Liu ME, Chang WC, et al. Sleep apnea and the risk of dementia: a population-based 5-year follow-up study in Taiwan. PLoS One 2013; 8: e78655.

4 Leng Y, McEvoy CT, Allen IE, et al. Association of sleep-disordered breathing with cognitive function and risk of cognitive impairment: a systematic review meta-analysis. JAMA Neurol 2017; 74: 1237-1245.

5 Jonker C, Geerlings MI, Schmand B. Are memory complaints predictive for dementia? A review of clinical and population-based studies. Int J Geriatr Psychiatry 2000; 15: 983-991.

6 Gifford KA, Liu D, Lu Z, et al. The source of cognitive complaints predicts diagnostic conversion differentially among nondemented older adults. Alzheimers Dement 2014; 10: 319-327.

7 Reisberg B, Shulman MB, Torossian C, et al. Outcome over seven years of healthy adults with and without subjective cognitive impairment. Alzheimers Dement 2010; 6: 11-24.

8 Mendonca MD, Alves L, Bugalho P. From subjective cognitive complaints to dementia: who is at risk? A systematic review. Am J Alzheimers Dis Other Demen 2016; 31: 105-114.

9 Vaessen TJ, Overeem S, Sitskoorn MM. Cognitive complaints in obstructive sleep apnea. Sleep Med Rev 2015; 19: 51-58.

10 Folstein MF, Folstein SE, McHugh PR. "Mini-mental state". A practical method for grading the cognitive state of patients for the clinician. J Psychiatr Res 1975; 12: 189-198.

11 Nasreddine ZS, Phillips NA, Bedirian V, et al. The Montreal Cognitive Assessment, MoCA: a brief screening tool for mild cognitive impairment. J Am Geriatr Soc 2005; 53: 695-699.

12 Hoops S, Nazem S, Siderowf AD, et al. Validity of the MoCA and MMSE in the detection of MCI and dementia in Parkinson disease. Neurology 2009; 73: 1738-1745.

13 Gagnon JF, Postuma RB, Joncas S, et al. The Montreal Cognitive Assessment: a screening tool for mild cognitive impairment in REM sleep behavior disorder. Mov Disord 2010; 25: 936-940.

14 Villeneuve S, Pepin V, Rahayel S, et al. Mild cognitive impairment in moderate to severe COPD: a preliminary study. Chest 2012; 142: 1516-1523.

15 Dalrymple-Alford JC, MacAskill MR, Nakas CT, et al. The MoCA: well-suited screen for cognitive impairment in Parkinson disease. Neurology 2010; 75: 1717-1725.

16 Trzepacz PT, Hochstetler H, Wang S, et al. Relationship between the Montreal Cognitive Assessment and Mini-Mental State Examination for assessment of mild cognitive impairment in older adults. BMC Geriatr 2015; 15: 107.

17 Dong Y, Lee WY, Basri NA, et al. The Montreal Cognitive Assessment is superior to the Mini-Mental State Examination in detecting patients at higher risk of dementia. Int Psychogeriatr 2012; 24: 1749-1755.

18 Ciesielska N, Sokolowski R, Mazur E, et al. Is the Montreal Cognitive Assessment (MoCA) test better suited than the Mini-Mental State Examination (MMSE) in mild cognitive impairment (MCI) detection among people aged over 60? Meta-analysis. Psychiatr Pol 2016; 50: 1039-1052.

19 Chen X, Zhang R, Xiao Y, et al. Reliability and validity of the Beijing version of the Montreal Cognitive Assessment in the evaluation of cognitive function of adult patients with OSAHS. PLoS One 2015; 10: e0132361.

20 Albert MS. Changes in cognition. Neurobiol Aging 2011; 32: Suppl. 1, S58-S63.

21 Gagnon K, Baril AA, Gagnon JF, et al. Cognitive impairment in obstructive sleep apnea. Pathol Biol 2014; 62: $233-240$.

22 Ferini-Strambi L, Baietto C, Di Gioia MR, et al. Cognitive dysfunction in patients with obstructive sleep apnea (OSA): partial reversibility after continuous positive airway pressure (CPAP). Brain Res Bull 2003; 61: 87-92.

23 Verstraeten E, Cluydts R. Executive control of attention in sleep apnea patients: theoretical concepts and methodological considerations. Sleep Med Rev 2004; 8: 257-267.

24 Rosenzweig I, Glasser M, Polsek D, et al. Sleep apnoea and the brain: a complex relationship. Lancet Respir Med 2015; 3: 404-414.

25 Gosselin N, De Beaumont L, Gagnon K, et al. BDNF Val66Met polymorphism interacts with sleep consolidation to predict ability to create new declarative memories. J Neurosci 2016; 36: 8390-8398.

26 Baril AA, Gagnon K, Arbour C, et al. Regional cerebral blood flow during wakeful rest in older subjects with mild to severe obstructive sleep apnea. Sleep 2015; 38: 1439-1449.

27 Baril AA, Gagnon K, Brayet P, et al. Gray matter hypertrophy and thickening with obstructive sleep apnea in middle-aged and older adults. Am J Respir Crit Care Med 2017; 195: 1509-1518. 
28 Strauss E, Sherman EM, Spreen O. A Compendium of Neuropsychological Tests: Administration, Norms, and Commentary. New York, Oxford University Press, 2006.

29 Petersen RC, Morris JC. Mild cognitive impairment as a clinical entity and treatment target. Arch Neurol 2005; 62: $1160-1163$.

30 Galasko D, Bennett D, Sano M, et al. An inventory to assess activities of daily living for clinical trials in Alzheimer's disease. The Alzheimer's Disease Cooperative Study. Alzheimer Dis Assoc Disord 1997; 11: Suppl. 2, S33-S39.

31 Sleep-related breathing disorders in adults: recommendations for syndrome definition and measurement techniques in clinical research. The Report of an American Academy of Sleep Medicine Task Force. Sleep 1999; 22: 667-689.

32 Hanley JA, McNeil BJ. A method of comparing the areas under receiver operating characteristic curves derived from the same cases. Radiology 1983; 148: 839-843.

33 Hosmer DW, Lemeshow S. Applied Logistic Regression. 2nd Edn. New York, John Wiley \& Sons, 2000

34 Cherbuin N, Reglade-Meslin C, Kumar R, et al. Risk factors of transition from normal cognition to mild cognitive disorder: the PATH through Life Study. Dement Geriatr Cogn Disord 2009; 28: 47-55.

35 Liguori C, Mercuri NB, Izzi F, et al. Obstructive sleep apnea is associated with early but possibly modifiable Alzheimer's disease biomarkers changes. Sleep 2017; 40: zsx011.

36 Julayanont P, Brousseau M, Chertkow H, et al. Montreal Cognitive Assessment Memory Index Score (MoCA-MIS) as a predictor of conversion from mild cognitive impairment to Alzheimer's disease. J Am Geriatr Soc 2014; 62: 679-684. 